\title{
Sfficinelle $\mathfrak{P}$ flanjen $\mathfrak{B} \mathfrak{r} \mathfrak{a}$ [ilienż,
}

von

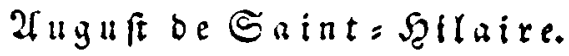

Fin 2ludzung and ben finf erften Lieferungen bes Franjoifitigen Sriginals, bearbeitet vim

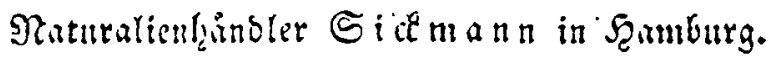

2) Strychnos Psezdochina. Hilair.

S. caule inermi tortuoso; cortice suberoso; foliis ovatis quintuplinerviis, subtus villosis; floribus racemoso-paniculatis, axillaribus, pedunculisque villosis.

Quina do Campo "); Braf. Shamen. Spreng. System. Vegetab. I. p. $672^{*}$ ). Hilaire Plantes usuelles Tab. $I$.

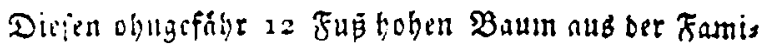
lie ber 2lpocineen findet man in bend sftiden Theile ber şro:

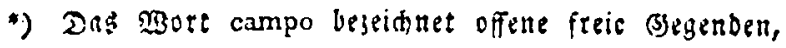

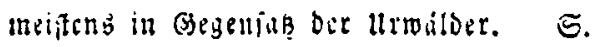

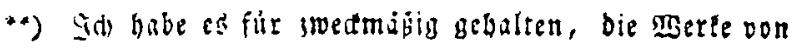
Midner und $\Xi$ waltes, fo wie von Gprengel, no es anging, zu cititen. E. 
Yroving Minas - Geraes, im Diamanten = Diftrift, in Det Buiften yon Goynz $2 c$.

Unter allen 2 rzmenpfanzen $\mathfrak{B r a f t i e n s}$ ift vielleidt die Guina do Campo am meiften bewaihrt geftmben uno im

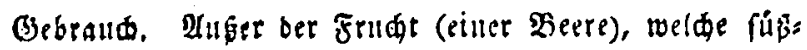
lid iff unb von findern gegeffen toito, haben alle Theile Diefer \$fanze einen feht bittern etwab adfringirenben befduace, der aber bauptridglid in ber Rinde liegt.

Diefer tortigen, andwendig meider und odergelben, iumenoig etwas bärtern und mebr granen Rinde bedienen fí aud bie Brafilier olngefábr in denfelben franthcis ten, gegen benen ill Europa sie Ehina angewandt wiro, vorjuglid aber in ben $\mathfrak{B C}$ felfiebern, oie in einigen Pro=

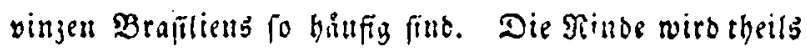
als Agfub, theils nle Wulver in Dofpn yon 0,001789 bis 0,003578 Silogi. gegeben. Eines der erfen Arrjte Brafilienb, Der vergleidjenve Berfude mit ber Guina do Campo und ocr פeruanifłen ådten Rinde

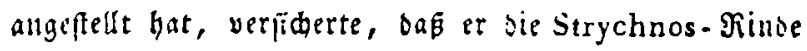
eben fo wirtam alo die adte Ehina gefunden babe. Die

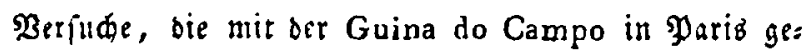
madt worben, faciuen obige Bebauprung ju beftatigen.

sad einer you $\mathfrak{B}$ a $u$ que $\mathrm{i}$ angeftelten Inalyfe entljält biefe Riube: 1) Einen bittern Etoff, ber ben

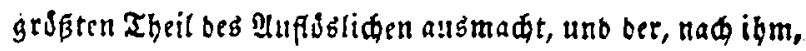
die Zieber vertreibende Rraft zu enthalten (ळeint. 2) Fine befonbere barjige Eubfanj, fehr auffistid in Illeobol 
von $36^{\circ}$, weniger in abfuluten. 3) Eilte gefärbte gums miartige Eubianj mit cinem thicrifien Stoff

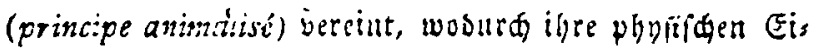
genidinftall mobificirt werbell. 4) Eine cigne Săure,

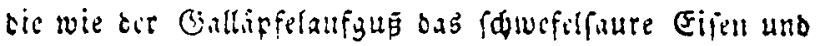
ben feim nicorrfdistigt, aber sies mit MRodificationen, welde nime criallen, biefe Giure mit ber Ballubfikure furr icentifith ju nehmen.

Inffallewo ifr es, dú ill der Riade des Strychn. Pseudochina wese: Ebinitit now Brucin, whe alte nidt eine

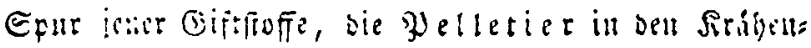
augen :c. sutdofte, anfgefunten. Celbft bis bittcre gु riucip oer Timoe in Thteradern eingeifrist, bradte teine bem

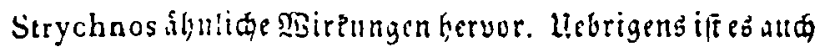
befumut, onf nibt alle Arten ven Strychnos (toie j. $\mathfrak{B}$. S. potatorum) giftige (Figenfáften haben, und das man

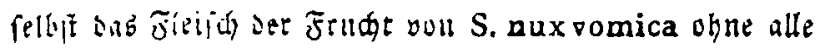
Gefabr eाা? Fann.

2) Cinchona ferruginea. Hilair.

C. frutescente, gracili, vix ramoso; foliis oblongo lanceolatis, subangustis, margine revolutis, superne profunde sulcatis, coriaceis; racemis elongatis interruptis.

Macrocnemum foliis oblongo-lanceolatis coriaceis integerrimis. Roem. et Schult. Syst. Veg. V. p. 6. Spreng. Syst. Vegetab. I. p. 764 . 
Quina de Serra; Quina de Remijo. Hilaire, Pl. usuell. Tab. II.

3) Cinclona Vebozii. Hil.

C. caule frutescente, gracili, vix ramoso; foliis ovatis, utrinque acuminatis, margine revolutis, superne profunde sulcatis, coriaceis; racemis axillaribus interruptis.

Macrocnemum foliis ternis, lato-lanceolatis, undatis, integerrimis, coriaceis. Roem. et Schult. Syst. Veg. V. p. 6. Spreng. Syst: Veget. I. p. 704 .

4) Cinchona Remijiancr. Hil.

C caule fiutescente, gracili, vix ramoso; foliis late ellipticis, obtusis, cuspidatis, in summum petiolum subdecurrentibus, margine revolutis, superne profunde sulcatis coriaceis; racemis axillaribus interruptis. Spreng. Syst. Vegetab. I. p. 704.

Sbige brei Shilla: Arten werben noll ben Eimroh=

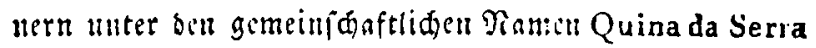
unb Quina da Remijo mit einander verwethfit. Senen

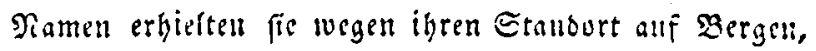
uno biefen, weil eilt SBmbarjt diefes Samens ifhen juerf

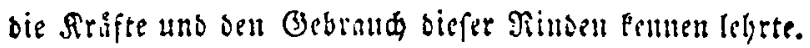

Die C. ferruginea fuwohl, wie bie beiben albern Iltten wadjen jivifden ber $17^{\circ} 50^{\prime}$ anb $21^{\circ} 45^{\prime}$ Elloers

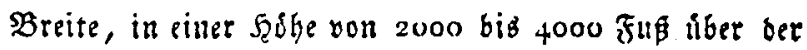
Diecresflude, auf den unfrudtbaten pablen Bipfel Det 
Berglette, welde fid yon Euten nab Norben in ter Probin; Minas erftrectl, nno bie sie Brimie ber Urwifts ber unb bes unberwaldeten ebenen Lantes bildet. 2Bo fie

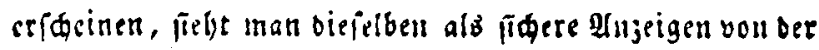
Gegemionat des Eifention.

Shre bitteren unb aditringirenden Rinben fommen an

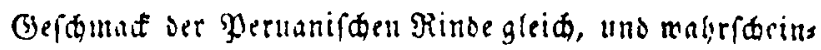
lite enthalten fie dicfelben Grmblioffe. Bou ben Biwohs Jlen jener Begrnoen werben jie mit ziemlid guten Erfolg zur Betinnpfung ber Firker nligewandot. Dbgleid sirfels

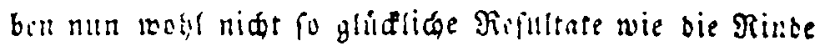
run Strychnos Pseudochina unb die Pirman = Rinbe lic: firn; fo Buncut fie oud als nuliguntes Surrogat anges wand wercen, vorjúglid oa in ben Gegenden, wo biefe brei Simqon:2artra wadjen, Str. Pseudochina nidt vorpủnent.

5) Exostema cuspidatum. Hilair.

E. foliis maguis lancenlata-ovatis, cuspidatip, nervosis, subtus villosis; parivula terminali; limbo calycino obscure quinquedentato, ovarin lnagiore; filamentis medio villosis; stigmate bifido.

Quina do mato. Spreng. Syst. Veget. 1. zo6. Hilaire Plant. usuell. T. III.

6) Exostema Australe. Hil.

E. foliis magnis, ovatis, nervosis, inferne subvillosis; panicula terminali, sessili, profunde tripartita; 
tita; limbo calycinn ovario multo hreviore, quinquelobo; staminibus glaberrimis; stigmate indivisa. Plantes usuelles Tab. III. b. Spreng. Syst. Veget. I. p. 106.

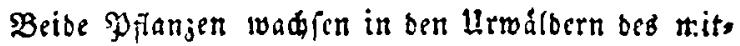
tagigen $\mathfrak{B}$ rafiliens, unb fo wenig ályntids fie einander fino, werben fie bod oft unter bem Nanten Quina do mato mit einanber verwedffelt.

Die Finbe ciefer briben Ejoftemen roird, in Ers mangelung mirfiancrer Mebifumente, glcidfallo in SBrofelfiebern gebraudt; abst meit whjpriger, woniger bitter und weniger zufatamenjielyeno, als sie orei áarea હindonen Srafitiens, fheintu ibre Fraffe jenen wit

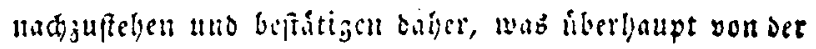
Soirffamkeit ber E;opemen órobnditet ifr.

7) Evodia febrifuga. Hilair.

E. caule arboreo; foliis ternatis; foliis lanceolato ellipticis, subacuminatis; panicula terminali, pubescente; nectario simplici; ovario muricato.

Ties follas vermellas. - Larangiera de mato. -

Quina. Plantes usuell. Tab. IV.

Ein bolyer $\mathfrak{B a u m}$ altb ber natúrliden Familie ber Rutaceen, oer in ben Bergluilbern ber Provillz oer פRis nen, vorjliglid in oer Míbe voll Habira-de-Matrodento iud

Die Rinde unb felbft bas Felj biefes $\mathfrak{B}_{\text {aumes finb }}$ febr bitter uno abfringizeno, uno man braudt erftere mit vies 
vielen Erfoly anftatt ber פ̧eruanifhen Fillbe. Die Seifo

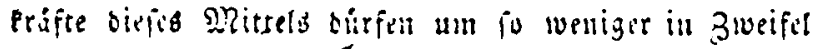

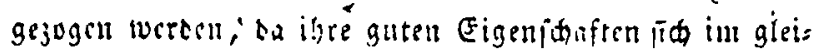
ditt Crabe in ser Minbe eines allsern Bames berfelken Familie, but betibnteu Cuspare (Cort. Angusturae. Bonplandia trifoliata Humb.) wiesirfinben, uno sie

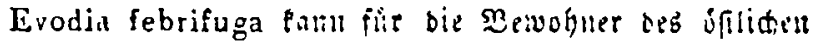
Theirs ber provillj Mlinas Geraes daś werbill, wab ble Angufura für sie Sowohner tes Drinopo's ift.

8) Sinnaritha versicolor. Hil.

S. foliis pinnatis; foliolis oblongo ellipticis; obtusissimis, retusis, nervo medio pubescente; panicula terminali laxa; floribus divisis decandis.

Paraëbo. Hil. Plant. usuell. Tah. V.

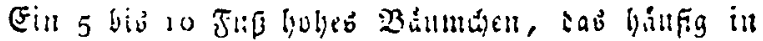

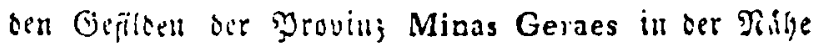
you Rio-de.S. Francisco (Certao) mádyit.

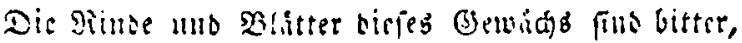
svic sie Simaruba amara teno librigell Arten oerfolben

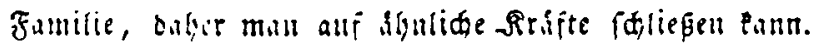

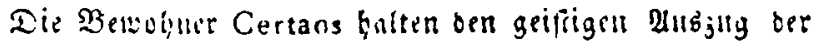

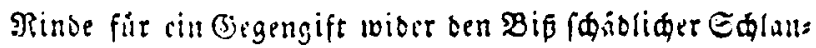
get. 2tud wiro fie mit bem griften Erfolg jur Szeilumg Ecr \&a uffut (maladie pediculaire) bei Menfa)ch,

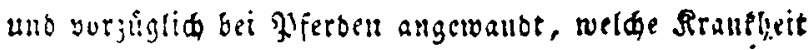
in iener Gorgeno fille báufig ilf. Durd folgended fattum bube id die Birefamesit diefer Yflanze gegen Ungejicfer 
beftatigt gefunden. In einer Fifie, bie eine große $\mathfrak{A n}_{z}$ aht

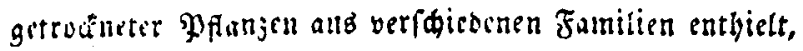
luarell 4 Exeluplare ber S. versicolor bigepaft. Alle biele Pllanjen wurben ourd cine grofe Drenge Laryen ei, nç গুinus zerfreffen und nut jene 4 Excmplare allein blicben unangeribrt. Bislleidt foute man, in oer Bors ausfeting duf mifere פplanje bell Birterfioff febr cons centriat unb nut wenige meblige Theile in jid enthalte, Diefe Erfteinung erfliren, alfeil warum waren it ber: felben Sire Ylanjenexemplare von weit bitterem Ges famac, wie j. $\mathfrak{3}$. Simaruba floribunda uno Evadia febrifuga, ganj jerfreffen? Wie bem aud feg, é bleibtau:

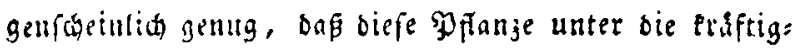
fen Mittil wiocr bas lingejiçer gehsirt. - Es iff bes Pannt, daßi die Simaruba amara (Quassia Simaruba) mit

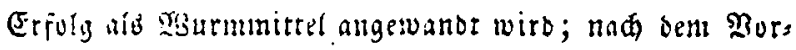

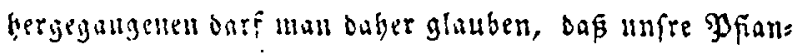

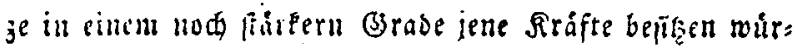
bel. Da einige Einuvolger Certaos biefe Pfianje aber fir giftig bolten, fo burfen bie Berjude mit oerfelben nut mit gehưriger Borfidt angeftellt werben; obgleid es wabrifeinlid ith, baj fie nur, wie die meiften bittern Srittel, burd) зu ftarle Gabe ober ourd falfde Innens buing den SRentben natitleilig geroorben.

9) Cephaelis Ipecacuanha. Rich.

C. caule simplici, saepius erecto; foliis lanceolato ovatis, acutis, basi acuminatis, integerrimis, XIV 3o. 1 seft. 
supra scabriusculis, suhtus subpubescentibus; ca. pitule terminali pedunculato, solitario, pedunculc pubescente; involucro 4-polyphyllo.

C. emetica Pers. Syaop. 1. p. 20? (exot. Syn. Linn. et Willd.)

C. Ipecacuanh. Spreng. Syst. Veg. I. 747. Hil. Pl. usuell. Tab. VI.

Callicocca Ipecacuanha. Brot.

Poaya; Poaya de mato; Poaya dz botica.

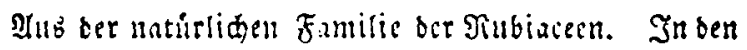

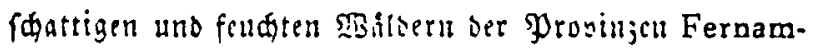
buco, Bahia, Esperito-Santo, Rio de Janeiro, Mi. nas, bib zllm 22 Brabe Ciber Breite.

Die erfte Entoectung ocr Ipecacuanba *) if ben In:

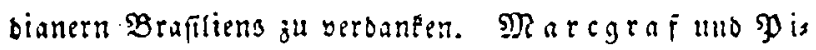
fon madten fie juerft in Europa befanut, unb Erfilys rung beftarigte bals bas $\mathfrak{L}_{0} b$, weldes fie siefom treffliden feilmittel beigelegt. Siacefpen ba ibre Defdreibung jeks, mangelhaft war, besiente man fid eiuer langen Reihe vout Jabren der Ipecacuanha, oine ju wiffen, won welder Y)fanze fie abftamme, bis entlid im Jabre 1800 der Doctor Antonio Bernarino Bones blúherbe Erems plare bavon nad Europa fante, wo Brotero fie bes farieb und je in ben Sdriften ber Simueifoen Societit ju Ronton abbitcen liep.

Der

*) Den bieier afgemein befnnlen Pfanje babe id nur bas, wab nell und mir weniger befannt bu feun foilu, in Der deberfesung wicbergegeben. $\varsigma$. 
Der Rame Ipecacuanha, ourd Rarcgraf unb $\mathfrak{P}$ i i on eingefíthrt, ift bei ben Braịliern felbet unbelannt; mut im Diffrift rolt Minas. Novas babe id ihn ciniges mal ucment bjren, obne jebod in Erfabrung zu bringen, welde Y)fanje bamit gemeint wurbe. Dagegen ift fie allgemein unter den Namen Poaya betannt, ein Name, ber nber nidt nur biejer, fonbern alld bell librigen Breden erregenben YYflanjen, bie alß Surrogate derfelben ges braude, gegeben wirb. Das $\mathfrak{B}$ ort Ipecacuanba fommt

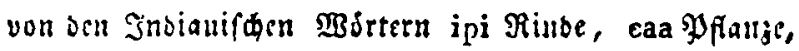
cua wohlriedend, nha geftecift (wohlriedende und ges

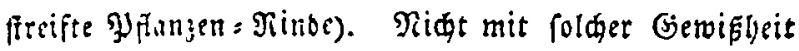

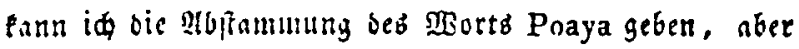

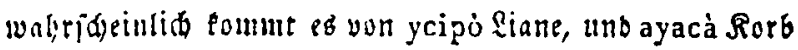
(Rine zum Sorbfechten), unb wurbe wohl zuerft einer Eqlimgplanje gezeben, deren șurjel $\mathfrak{B r e d e n}$ erregenb ift, uns bie id ill ber Jolge befwreiben werbe.

Die Ralffute, welde die $\mathfrak{B u r}$ el alls dem Jnnern bejiérall, wiffen fie redt gut yon andern $\mathfrak{B u r z e l n ~ z u ~ u n s ~}$

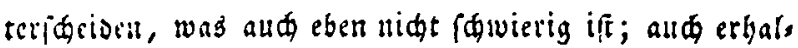

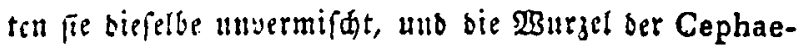
lis toirb jetzt yon Rio nad Europa gefandt. Es ift nidt Der Fall, daßj man die $\mathfrak{B}$ urjell yon Jonidium Ipecacuanha, bie wentig hânig yorfommt, uns bie von Jonidium parviflorum, weláe weit entfernt von ber Cephae.

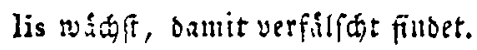

Sbyleić man dic Ceph. Ipec. in ber Räbe yon Rio $6^{*}$ uno 
unb allbern gropen Gtibten bxinabe ginzlid auggerottet, fo ift fie bod nud an vielen S:ten fihr hallig. 2uber ba man fie ohue Norficht altoreipt, man midt bie Reife ber

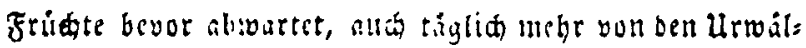
berll allggerottet wiro, fo wirs jê wohl mit ser geit fels

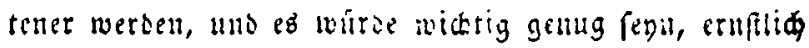
an ben Anbau berfelben ju benten.

10) Riciardasonia rosea. Hilair.

R. caulibus apice praecipue molliter hirsutissimis, foliis ovatis vel ovato-ellipticis, acutis, margine praecipue scabris, per paria distantibus; stipulis ultra medium divisis; corollae laciniis pilosis (toribus roseis).

Pnaya do Campo. Hil. Pl. usuell. Tab. VII.

Eine ausbauernbe Erautarrige \$̧Panje allb ber Familie ber Rubiaccen, bie in oen bitern und unbewalseten Thei: len yon Comarcas, S-Joao-del-Rey mo Villa-Rica, befonberd am Naube ter $\mathfrak{B}$ sge utb an befugten Drten pebr gemein iff.

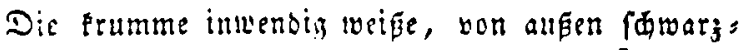

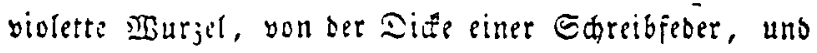
von einem ter Ipecacuanha fejr ähnliden Bepdimad, wirb yon ben Bewolnern der Ungebung von Joao-delRey mit vielem Erfolg als Eubiritut der Ipecacuanba

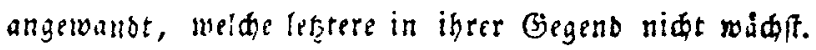
Shre Firifte fillo nidt mur der wabten Ipecacuanha vol, lig glitid, fonterm man erbilt gar nod mit Eleinern Bas 


\section{$-85$}

ben gleide Refultute. Seţt, da bie Cephaelis Ipecacuanha feltener wird, funnten wrälier wohl ben Bers

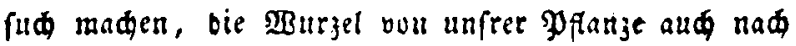
Europa zu fenden, beren Brandbarkeit man gewis balo anerfennen wirbe. Sic wirbe aud weit leidter zubauen feyn, ba fie fid mit einer allserorbentliden feidtigpeit vermebrt; fie hat friucn Edatten wie jen: nsthig, uns gebeight redt $g$ t in eineu wenig frudtbaren Boben.

11) Richardsonia scabra. Linn.

R. caulibus pilosis; foliis ovatis ovatove- lanceolatis, rariis oblongis, aculiusculis, margine praecipue scabris; stipulis usque ad medium non divisis; corollae laciniis apice pilosis (floribus albis).

R. pilosa Ruiz et Pavon - R. brasiliensis Gomes.

Spermacoce hexandra Richard.

Ipecacuanha amilacé ou blanc Dict. medic. Plant. usuell. Tah. VIII.

Poaya do campo.

Sebr gemein in ber গyrovin; Rio de Janeiro, an

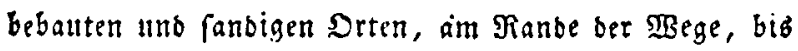
felbft $i$ ben wenig begangenen Strafien von Rio fineill.

Die $\mathfrak{B}$ urzel ift 1 bis 7 Soll lang, etwad búnner als eine Shreibfeber, einfab ober iffig, oft gef rummt; balb ohne bald mit geringelten 5 ffern, bie gewshnlid breiter als oie oer wahren Ipecacuanha fint; igre im frifden Zuftante weī̄e Farbe wiro burds Troünen afdgrau;

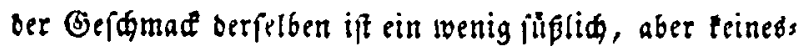




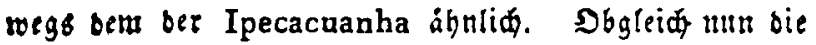
2Burjel nidt ganz bie heroifden Eigen/daften ber yor: hergebenden bejist, fo tum jie dod nothigenfalls anftatt ber Ipec. gebraudt werben. Mant madet zrat yon ibr in Rio wenig Bebratla, bies rilget aber unt daber, weil

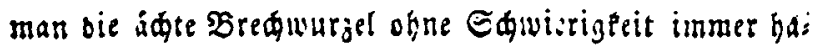
ben paltr.

12) Fुoniäum Poaya. Hilair.

J. hirsutissimum; caule suffruticoso, saepius simplici; foliis alternis, subsessilibus, ovatis, basi Eubcordatis, aeutiusculis obsolete dentatis; stipulis linearibus, integerrimis, vix manifestis; laciniis calycinis integris; petalo inferiore maximo, late obcordato; filamentis apice extus barbatis; antherarum processu membranaceo minimo. Poaya do campo. Hil. Pl. usuelles Tab. IX.

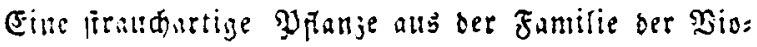
lacell, hiufin im weftiden Thcil ber Provinz Rio de St. Fraucisca, in ben PDrovinjen Minas. Geraes, Goyazetc.

Die Eimughner obiger Jrovinjen bedieneu fid der BBurjeln biefer $\mathfrak{O}$ fanme mit Erfolg, um bie Ipecacnanha

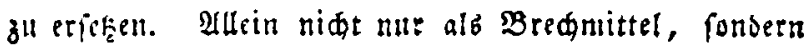
auc a!d Laxans wirb biefelbe, indem man antere Dofen nimunt, altgetonnbt. Dieje Pflalize befatigt íbrigens bie Breden erregenbe Birpung, bie man suben Biolaceen wahrgenommen, uno wollte man einige Berfude anfels leil, fo wirbe man biefe Eigenfdaften, in cinem mehe 
ober minbern Grabe, in ben jablreiden Arten bicjer Fas milie in ben verfdiebenen $\mathfrak{P r o v i m z e n ~} \mathfrak{B r a f i l i e n s}$ wieber: fincen.

13) Conohoria Lobolobo. Hilair PI. usuell. Tab. X. und

14) Conohoria castanefolia. Hil. Meftige Stralldgerwidge aus ber Familie ber Bios laceen.

(Die Blitter von beisen $\mathfrak{Y}$ fanjen, bie gefodt in einigen (3egenden ber $\mathfrak{P}$ rovili Rio von Regern genoffen worben, empfieblt $\sigma$ t. F̧ilaite jum Inbau als (je: múfep flanzen).

15) Эัonidium Ipecacuanka Ventenant.

J. foliis alternis, lanceolata-ovatis, serratis utrinque acutis; stipulis acuminatis, membranaceis, medio nervosis; calycinis divisuris sempinatifidis; petalo inferiore maximo; transverso elliptico.

Viola Calceolaria et Ipecacuanha Lin. - Viola Itoubou. Aabl.

Pombalia Ipecacuanha. Vandellii. - Jonidium Itubu. Humb. et Kunth.

Poaya, Poaya da praya, Poaya branca. Pl, usuell. Tab. XI. Solea Ipecacuanha Spreng. Syst. I. 804 . Eine Frautartige peremirende $\mathfrak{P f l a n j e , ~ m i t ~ b i u n e n ~}$ aufiteigenden ober nieberliegenden etwab.holgigen Gten: geln. Sie wdd/t am bdufigften ju Cayenne (nแ⿰ it. Peru), 
Pera), bod findet fie fíd bann uno wann an ber Rúfte Brafitiens yom 2Amajonenftrome bis jum Cap Frio.

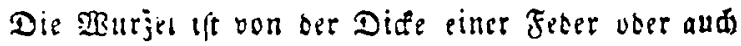
etwas ffirfer, mebr oter weniyer frumm, etwas geftreift ober riling, imbendig weiß, von allsen reiflid: grall, am Ence mit farten uno jablreiden Fafern befert, yon fabem uto unungenelmen (selfmaf.

Angenein bepantu find cie Bredon cregente Figens f Diefelbe als bis befe freilmittef wiber oie Tilbr; alld be: bauptetell etlica Eimwobner der Provinz Rio-Grandedo-Norte, oag, um ous Poongra grindlid ju heilen, cs binreident wite, einige Tage lang eine leidte Mtfodung

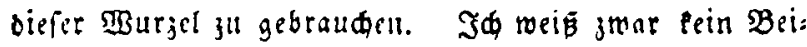
fpiel, meides lestere Bebauptung befititigt, aber fie

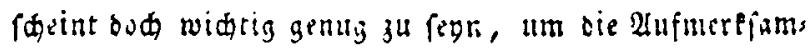
Peit oer Mlerjte ju verdiemen.

16) Spermacoce Poaya. Hilair.

S. glakerrima; foliis oblonga ellipticis, lascobtusis, apice acutis; stipulis setaceo multifidis; floribus capitatis verticellalisque; stamibibus exsertis. Розуа do Campo. Pl. usuell. Tab. XII. Tatirliàe Fumilie oer Pubiaceen.

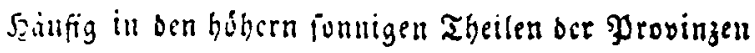
Minas Geraes und St. Paul.

In beiben Provinzen werbeu die $2 B u r z e i n$ anfratt ber Ipecacuanha gebratudr. Die $\mathfrak{B}$ ldtter find bon einen 
anfange pehr filien, nather aber fauren (S)efdmad, und wersen aló gatudung gegen Sutie und andere innere Edimerjen gebrauds.

17) Spernacoce ferruginea. Hil.

S: caule pubescente; foliis lanceolatis vel lanceolato-ovatis, lanceolatove-oblongis, lineatis subpubescentihus; floribus capitatis verticillatisque; involucro 6-8 phyllo; bracteis setaceo-multipartitis; staminithus exsertis.

Poaje. Puaja da Praya. Pl. usuell. Tab. XIJI.

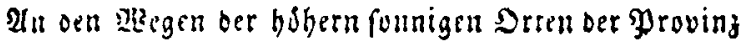
Minas unb St. Paul.

Soldoul diefe Pfanje nidt felten iff, fo viro jie bod nur ju Cap-Frio ampatt der I pecacuanha Bobralld ons youl gemadi uno da die guten Eigenfdaften berfelben gringut. Nitip ber Spermaroce Poayo ter Richardsonia rosea uno $R$. scabratephitigt diefalte bie Bredenters regenben Srufite der zweifamigen Rubiaceen.

18.) Calyptranthes aromatica. Hil.

C. frutescen's, glaberrima; foliis cannatis, magnis, oblongo-ellipticis; paniculis terminalibus axillaribuave, geminis.

Craveiro da terra. Pl. usuell. Tab. XIV.

Fin 8 big g $F_{41}$ hoher Etraud and ber Fomilie ber Ryrtaceen, in belr urrodibern ber Provin; Rio.

Die Blumentnorpen diefer Pllanje baben ben Be: rud 
ruch unb Befdinat ber Bervirjnelfen. Dogleid mun siefalben nidt villig fo ftare toie die jente frub, fo erbals

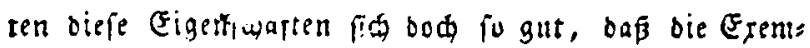
plare nucines Sterbarimm, obgleid fie bell Dampf oes

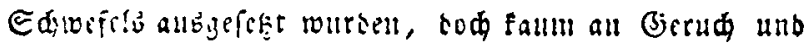
Gejdmack rerlusen butten.

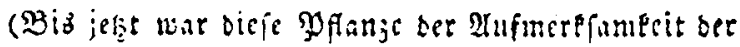
Bruftice entgangen. Fecr von $E_{t} \sqrt{2}$ ila ire glaubt,

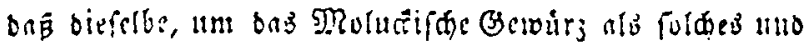
alb $\mathfrak{A}$ rgneyinttel ju erfegen, in ber Folje sin widtiger

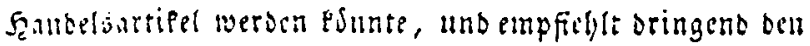
Inbau berfilbin. E.)

19) Diosera communis. Hil.

Pl. usuell. Tab. XV.

(F̧err von $S$ t. Feilaite warntoie Bielyjugttreis benben Einivoluer Brajifiend vor bicie und andere Drosera-Arten, die in ben Simpjon haufig wadien, inben fie din Edanifon febr madrbeifig finb).

20) Ticorea febrifuga. Hil.

T. caule saepius arhoreo; foliis ternatis; foliolis lanceolatis, acuminatis; in petiolum attenuatis; paniculis coarctatis; staminibus $3-6$ sterilibus.

Quina. Tres follas brancas. Pl. usuell. Tab. XVI. Diefe balo. mur frraldige, bald ald ein hober Baum werdente Sutncee wadf im sefliden Theil ber Pros yinj Minas. 
Die Sirifte ber fige bittern unb abfringitenben Niube tomena mit ber ber Evodia febrifuga Nr. 7 .

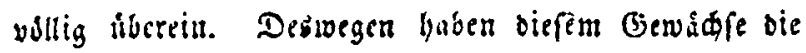
Finwohner obiger Probinz sen Ramen Quina beigelegt; die $\mathfrak{B e n e m u n g}$ Tres follas fúbrt ea wegen feiner brei Shatter, uno bus Bitwort brancas (weißi), um unfre Pflanje yon ber Evodia febrifuga zu unterf́feiben, bie rithlide Blitter bat.

21) Hortia Brasiliana. Velloz.

H. suffruticosa, subsimplex glaberrima; foliis sparsis exstipulaceis, integerrimis, punctato-pellucidis; floribus cymosis, terminalibus, roseis; pedicellis bracteolatis. - Pl. usuelies Tab. XVII.

Quina.

Ratúrlide Famifie oet $\Re$ litaceen. In ser Provinj Minas utto Goyaz.

Die Fieber yertreibense firaft ibrer Rinbe hat siefe Pflanze, fo wie viele andern, ben Ramen Quina jul ver: banten. Dod darf bieflbe nur alb ein überflifingeb Clrs: rognt angejeben werden, on Strychnos Pseudochina ill benjelben Begenden wie biêfe झflanje vortommt.

ع2) Euphorbia popillosa. Hil.

E. glauca; fol caulinis, oblongis vel oblongo linearibus, mucronulatis, integerrimis, glaherrimis; umbella aápius 5-fida, omnino papilloso-pubescehte; involucris sub 5 -gonis, intus lineatim 
villosis; divisuris 5 , erectis, semiovatis, obtusis, dentatis; 4 patulis transverse subellipticis; floribus masculis 25 , in fasciculos 5 dispositis, cum fasciculis totidem bractearum lanatarum alternan. tes.

Lecteira, Lechetres. Plantes usuell. Tab. XVIII.

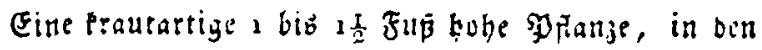
fonnigen Gefilsen oer $\mathfrak{P r u v i n z}$ Santa. Catharina unb Rio-grande do.sul.

Die Bewohner dicfer Drobinzen brinden fie als

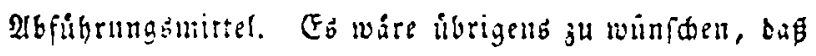

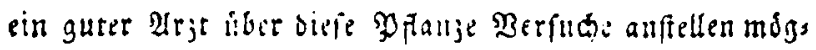
te, um allsjllnittein, in welder Ojabe fie bei reridietes un IIter genummen weroen mug, ba fie yon ben Eins

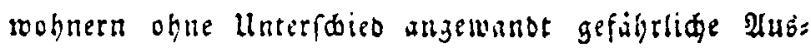
leerungen verurjadyen paun.

23) Anchieta salutaris. Hil.

A. suffruticosa, foliis alternis petiolatis; stipulis geminis, lateralibus caducis; floribus axillaribus racemoso - fusciculatis. Pl. usuelles Tab. XIX.

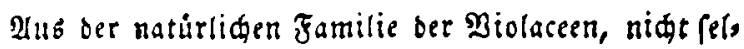
ten in bre Ungesung yon Rio de Janeiro.

Bon mef̧reren Eolunijlen ber umgebung Rio's toers

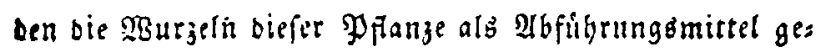
braudt. Wieit mehr Ifufmertfamete verbient bies Ges wåd aber wohl, oa man ihr bie Eigenidaften Şaut: Frantheiten 3 beilen jufdreibt. Es ift betannt, baja man 
bet ans *) eine Beitlang glaubte, eitte einheimifde Bioe lucee, bie Viola tricolor, esume alo sin ficherió reinigens beb Mittel angewanot werben. MRentr uhil bie Anchieta das Aufihen tincr Viola hátte, fo birrite man glauben, Die Yortugiefen batten in berfulben Friffte voratsogefeçt, welde in emer Viola ithes Baterlandes nurbandell feyn

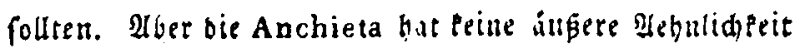

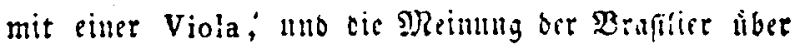
erfiere Eam aliu nur bas Refultat der Erfahrmag fion. Intem nun dieje $\mathfrak{Y}$ fanje die reinizenten Eigenfdaftin ber Bivlaceen alfí nette beffitigten, fo burfte eg, meince

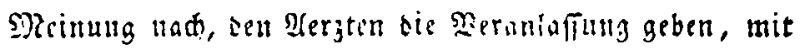
unjern cinbeimifgen Sivlacen newe ふerfude aujufrellen.

24) Fonidium parviflorum. Ventenant.

J. pubescens (saltem in brasiliensibus speciminibus) ; caulibus suffruticulosis, adscendentibus, debilibus; foliis inferioribus oppositis, caulinis alternis, omnibus serratis, basi acutis, stipulis brevibus, linearibus, acutis, integerrimis; floribus parvis; petalo inferiore late obcordato. - $\mathrm{Pl}$. usuelles Tab. XX.

Viola parviflora L. Solea parviflora Spreng. Syst. veget. I. 804 .

In ben mittágigen ßriben ber Proving St. Paul, bie Campos. Geraes genanut werden.

Bon

*) Das beist in Frantreid; denn in Teutidlunb witb an viclen Diten bie Viola tricolor mit Erfolg gegen genanute Sranfleiten angewandt. $\subseteq$. 
Bon ben Finmolncrn bes ipariffen Ifmerifad wirb

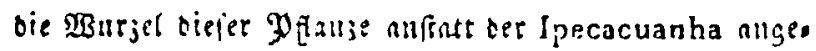

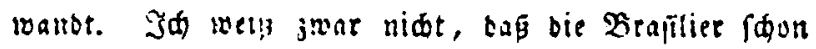

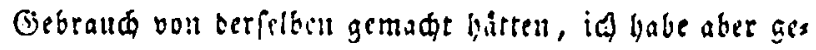
glatbe, ifinen ben ocrfelben anjeigen zu miffen, weil malt in jener Bryend der Yrovin; St. Paul weber die idie Ipecacuanha, nod eime ocr anbern Brajilianif rogate fincer.

25) Solanum Pseado-China. Hilair.

S. caule arboreo, inermi ; foliis lanceolato-oblongis, angustis, acutis, integerrimis, supra glabris, subtus in axillis nervorum fasciculatim villosis; racemis extraalaribus, hrevibus, monoolygocarpis; calycibus globris. - Pl. usuelles Tab. XXI.

Quina.

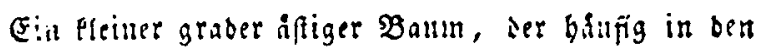
Szoljungen bes Dijrtifta Curitiba with/t.

Durd ibre Priftige Fieber verstibunbe Eigemidaf: ten if biefe Pranje eine bet widatiglen fúr ben allper

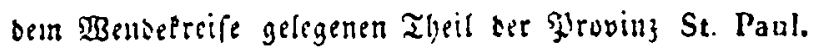
Jitre febr bittere, binute, beimabe glatte, bellgetbe unib

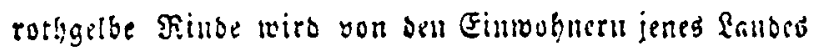
mit ben glictidiffen Erfolg wiber Das Fieber angewand, und jie laffen fich nidt yon ibrem Slanben, baj ibre Plans

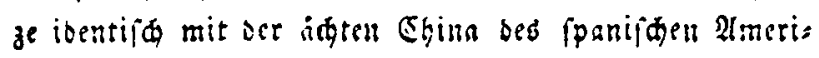
tab fey, abiringen. 


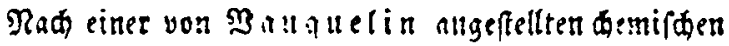
unterfudung eutgêlt die Siinse von Salanum Pseudn-

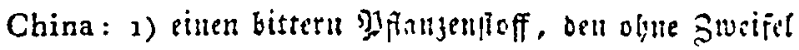

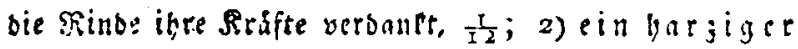
oder halbbarziget Etoff (matiere resireuse ou resinoide), ber cin wenig in saffer lisbar uno liter ip, 万丂 ; 3) eine Elcine Quantiatt eines iettige u f febris gen Stoffs (matiere visquese grassie); f) sime thies

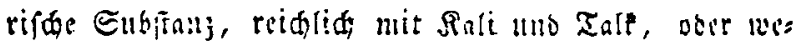
nigitens mit Gubmalaten vereinigt, welde baler alenlifd

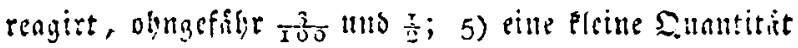

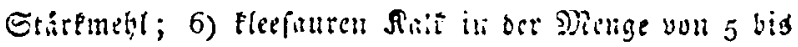
6 procent; 2) einen altbeill taltigten Eroff, set wonig

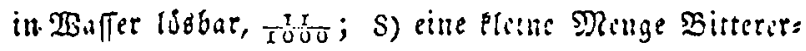

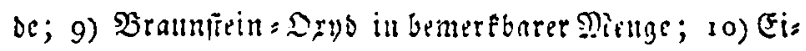
(enorno; 11) eine lige geringe Danntitit plobphorfaus

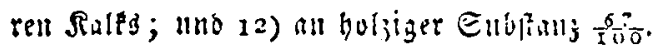

Bemertungsumerth if es nad ier $\mathfrak{B c o b a c i t u m g} \mathfrak{B} a$ us

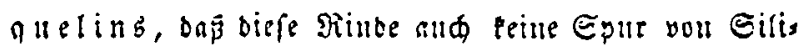
cium enthalt, on man bis seịt nod Eeine Pranje unters fudt, worin man uidt wenigftens etwas you dicfem Eroffe aufgefunoen.

\section{6) Davilla rugosc. Poiret.}

D. caule scandente, ramulis hirsutis ; foliolis oblongis, remotissime obsoleteque serratis, supra asperis, glabris, subtus nervo medio lateralibusque hirsuto-villosis; petiolo subtas villosissimo; pedun- 


\section{$-9^{6}-$}

culo pedicellieque hirsutis; petalis 2-3; pistillo unico. - Pl. usuell. Tab. XXIl.

D. brasiliaaa Decand.

Cipo de Carijó in ten Provillzen Rio de Janeiro uns Minas Geraes; Cambachinha jll Minas. Geraes; ripo de Caborln in aer Jyoving St. Paul.

Eine Kiane ans sir Jamilie ser Dillunassen, buflfig ill bell Urw.slsern Brafitiens.

Dits Orwido miro Cipo de Carijd, o. h. Siane Det

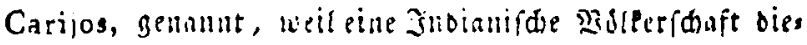

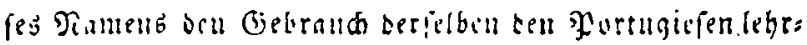
te. Die sisterter Cipó de Cahoclo knben uhugefibr bies

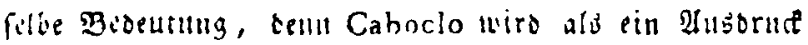

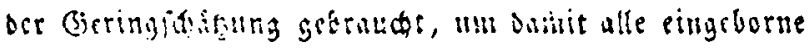
Jntinuifde Etámme jut hejpiđanen. Câmbä̈binha ents

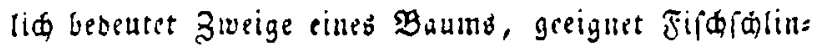
gen jul muden.

Man hat ifoul friber an ben Dilleniaseen abfiringi= rende Rrafie gifunden. Der berbe Gefdimaç ocerer Davilla fübrt don Beweis, oás fie ben adjringirenden Etuff mit den ubrigen $\mathfrak{P}$ fanjen iffer Fomilie theilt. Die Brapilier maden baģer cine glituliqe Intenbung mit birfer Pflanje, indem fie diefelben als Fomentation gegen $\mathfrak{A n}$ idivellung ber Beine two froben gebrautien, eine has, fige seantbeit in oem beisen und fellden abeile ifyes Ennter.

Eonft liffern ifge felor bieglamen atoeige nod treffs liqe 
fide Binder. Bie sle Rorbweide behanbelt wirbe aud fie gleide Dicufie thun rsment.

\section{7) Davilla elliptica.}

D. ramulis hirtellis; foliis ellipticis, utrinque obtusissimis integerrimis, crustaceo-coreaceis, supra scabris et glabris, subtus pubescentibus reticulatimque venosis; petiolo subtus villoso; racemis hirsuto-villosis, bracteolatis; calycibus sericeiis; petalis $1-6$ sub obcordatis; pistillis geminis. Pl. usuell. Tab. XXIII.

Cambaïbinha.

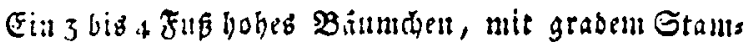
ane, in ben Difrift Minas - Novas.

Dbjltid bieje Davilla nide ju oenferben Sennomi:

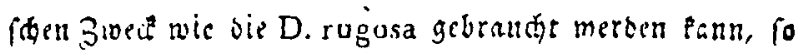
bat fie fid wegen ibrer Melyntideeit mit jenter gleiden Dameu ju verdanËn.

S3iro in den Biezenden, wo fie yorkommt, ald Butuntmit, tel allgersust, und ba fie all abfiringireno ift, madt

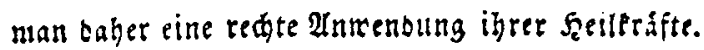

88) Curatella Cambaïba. Hil.

C. ramulis tomentosis; foliis ellipticis, latis, obtusis, plas minus rotundo-dentatis, supra scabris pilisque stellatis compersis, subtus tomentosis; racemis lateralibus, compositis; pedicellis calycibusXIV $\mathfrak{B 0}, 15 \mathrm{fft}$. 
que villosis; petalis valde caducis: - Pl. usuelles Tab. XXIV.

Cambasba.

Fin fleiner ober mittler frummer $\mathfrak{P}^{2}$ aum alls ber Eas milie oer Dilleniacten im metlliagen Theil der $\mathfrak{M r o v i n}$ Minas, ber Certao genamut wiro.

Stt Furopa verben bie sisunden oft mit eimer 2lbes dung von Cbiua getwafben, im Difript Ceriao madt man von der imnern Finfe der Caratella cinen áfnliden Bebraud. Fine glílifiche $\mathfrak{A}_{1 ! n}$ eneung oce nofiringiren: ben Etoff3, uns folylid fitmin wir ben Brafliern tie Fortiegung biefos Mrittelo, weltheo nur ourd Sufull bei ibuen eingefilbrt feyu wirb, felyr anempfeblen.

29) Echium plantugineum. Linn.

E. caule molliter hispido; ioliis radicalibus ovatis oblongisve, seu lineari-oblongis; superioribus linearibus, basi cordato-auriculatis; floralibus cordato auriculatis, falcatis; omnibus villosis; corolla calyce triplo longiore.

E. honariense Poir. Echium plantagineum et plantaginoides. Roem. et Schult. Spreng. Syst. Veget. I. 554 .

Burracha chimarona. - Pl. usuell. Tab. XXV. Diefe Boraginee, uriprüngliф im füblidaen Europa, Norcajrifa einbeimtju, ift wabrideinlid mit Sdmereien in cie feicer ron Rio de la Plato eingemantert. 3u 3ris ten couneri 
ben Etrinen bei Monte Video, nber iekt wie cille Menge auterer Europdifden Mflanjen hat iie fin binfig ourd BBrafilien nufer bem Siscubereife verbrettet.

Die Botagineen init troctnen Fridten fino wie bes lannt in Mllgemeinen erweidheno unb fdleimig. Die Colo: nifien ber Provin; Cisplatine fillben biefe Eigenfaaften in obiger Specics wiecpr, und reenten ciejelbe in shnli,

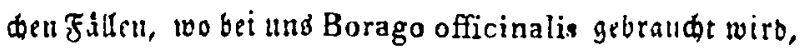
nu, unb fie baben ibe relbit den Ramen Burracha chimarona (miloce Borerid) beigelegt.

Páthere Prifung veid)iedemer in fublidjen

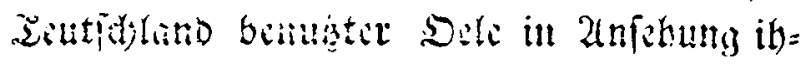

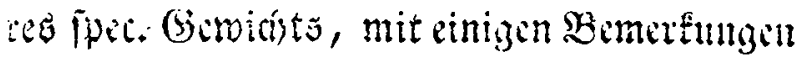

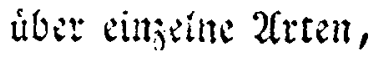

\author{
von \\ Profiffor Dr. Sdiblex in Thimien.
}

Э由 theilte in ben Inmertungen ju E hap ta l's 2 gris

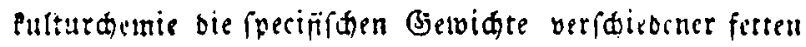
Delarten mit, aneldhe in fildiden Teutidant mehr in

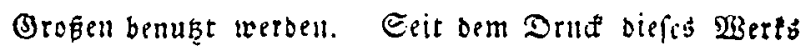
fano it Orelegenfyeit, nod einige alloere Mrteu ju unter,

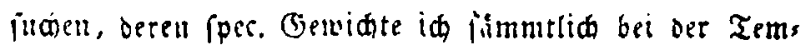

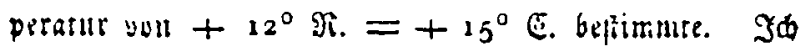

